\title{
Modelling and Practical Implementation of 2-Coil Wireless Power Transfer Systems
}

\author{
Hong Zhou, Bin Zhu, Wenshan Hu, Zhiwei Liu, and Xingran Gao \\ Department of Automation, School of Power and Mechanical Engineering, Wuhan University, Wuhan 430072, China \\ Correspondence should be addressed to Wenshan Hu; wenshan.hu@whu.edu.cn
}

Received 4 April 2014; Revised 8 August 2014; Accepted 21 August 2014; Published 3 September 2014

Academic Editor: Adam Panagos

Copyright ( 2014 Hong Zhou et al. This is an open access article distributed under the Creative Commons Attribution License, which permits unrestricted use, distribution, and reproduction in any medium, provided the original work is properly cited.

\begin{abstract}
Wireless power transfer (WPT) based on inductive coupling could be potentially applied in many practical applications. It has attracted a lot of research interests in the last few years. In this paper, the modelling, design, and implementation of a 2-coil WPT system are represented. The prototype system can be implemented using conventional power electronic devices such as MOSFETs with very low costs as it works in relative low frequency range (less than $1 \mathrm{MHz}$ ). In order to find out about the optimal working area for the WPT system, the circuit model based on the practical parameters from the prototype is built. The relationships between the exciting frequency, coupling, and output power are analyzed based on the circuit and magnetic principles. Apart from the theoretic study, the detailed implementation of the WPT prototype including the coil design, digital frequency generation, and high frequency power electronics is also introduced in this paper. Experiments are conducted to verify the effectiveness of the circuit analysis. By carefully tuning the circuit parameters, the prototype is able to deliver $20 \mathrm{~W}$ power through 2.2 meter distance with $20-30 \%$ efficiency.
\end{abstract}

\section{Introduction}

Wireless power transfer (WPT) technology can be potentially used in many industrial applications in case that the interconnecting wires are inconvenient, dangerous, or even impossible. It has attracted much of research attention in the last few years. There are several WPT techniques being researched presently. Far-field techniques use propagating electromagnetic waves that transfer energy the same way radios transmit signals but with much bigger transmission power [1]. Inductive coupling (or near-field) techniques operate at distances less than a wavelength of the signal being transmitted, which operates at a relative low frequency comparing with the far-field methods [2].

The early research and analysis of inductive coupling $[3,4]$ are mainly based on the pure physical theories, which could be difficult for the researchers from electrical engineering background to fully understand. Recent researches [5-7] start to use circuit and magnetic theory to study the fundamental principle of the magnetic resonance coupling, which makes the technology more tangible for a wider range of researchers especially for electrical and electronics engineers.

Potentially, inductive coupling WPT systems can be used in many industrial applications, such as power supplies for moving sensors and transducers [8], medical implants $[9,10]$, and wireless electric car charging stations [11].

In power industry, due to the importance of high voltage (HV) cables in the transmission network, on-site condition monitoring is a very important issue $[12,13]$. However, the power supply is always a major problem for the reliability of the monitoring devices which operate in the harsh outdoor environment with no reliable power sources. Some applications try to use the solar and wind power to solve the problem, but these power sources are not guaranteed in case of continuous bad weather condition which could occur frequently in some places like the mountain areas in South West China.

However, using the WPT technology, a tiny amount of the power can be harvested from the magnetic field around the HV power cables using induction coils and then transferred through the insulator (a few meters distance) to power 
the monitoring devices installed on the towers, which could be the potential solution for the problem. For this kind of applications, the key requirement is to deliver sufficient power to the load with relative high power transfer efficiency (PTE) and considerable distance. Robustness and reliabilities are also important issues especially for case of the HV cables monitoring.

There are many configurations of WPT system developed recently including the 2-coil [14, 15], 3-coil [16], and 4-coil $[17,18]$ links. The multicoil structures are able to increase the PTE in some cases, but the price is the increasing complexity of the system structure. Each coil has its own parameters such as resistance, inductance, and capacitance. Some parameters may be sensitive to the external environment like temperature and humidity. More coil loops mean that more tuning work is required to get all the parameters aligned. It could be difficult to maintain the optimal performance in some harsh environment for the practical applications. Balancing the performance, complexity, and reliability, the simple 2coil structure may be the best option for some industrial applications.

In [14], the optimum design of an inductively coupled 2-coil power transfer system is studied using the conjugate image impendence theory [15]. Theoretically, the optimum parameters of WPT systems can be calculated to maximum power transfer. However, in some applications, it could be difficult or costly to change the intrinsic circuit parameters, which is required by the theory.

In this paper, a WPT solution could be potentially used for HV power cable monitoring is introduced. In order to optimize the working conditions, the circuit models with the practical parameters are analyzed and simulated. Based on the theoretical study, a WPT prototype designed for practical $\mathrm{HV}$ power cable monitoring is set up. It is able to deliver $20 \mathrm{~W}$ power for a distance of $2.2 \mathrm{~m}$. As it is designed for the practical applications, the resonant frequency is reduced to several hundred kilohertz, which are within the ranges of conventional power electronics components such MOSFETs. It can be easily commercialized as all the power electric components can be purchased with low costs.

\section{Circuit and Magnetic Principle of 2-Coil WPT Systems}

2.1. Optimal Circuit Model. Figure 1 shows the equivalent circuit model of the 2-coil WPT system using magnetically coupled resonator, where the coil system with self-inductance $L_{1}$ and $L_{2}$, mutual inductance $M$, and copper loss $r_{1}$ and $r_{2}$. As both coils are air core inductors, the iron loss is not considered. Both $\mathrm{Tx}$ and $\mathrm{Rx}$ coils are connected to the resonant capacitors $C_{1}$ and $C_{2} . R_{c 1}$ and $R_{c 2}$ are the parasitic resistance of $C_{1}$ and $C_{2} . r_{p}$ is the impendence of the AC power source. $R_{L}$ is the load resistance of the output.

For the system shown in Figure 1, the optimal parameters which maximize the wireless power transfer are analyzed in

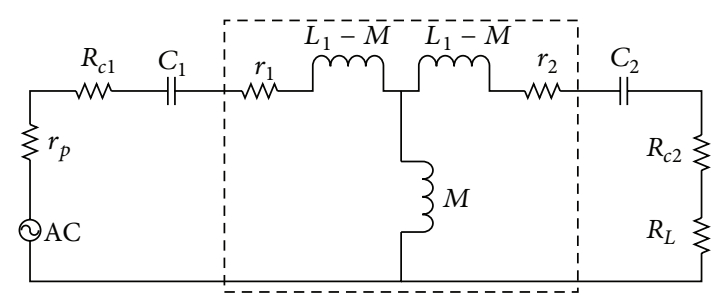

FIGURE 1: Equivalent circuit model of two-coil WPT system.

TABLE 1: Circuit parameters.

\begin{tabular}{lc}
\hline Parameter & Value \\
\hline$L_{1}, L_{2}$ & $298 \mathrm{uH}$ \\
$C_{1}, C_{2}$ & $200 \mathrm{pF}$ \\
$r_{1}, r_{2}$ & $2 \Omega$ \\
$r_{p}$ & $2 \Omega$ \\
$R_{c 1}, R_{c 2}$ & $3 \Omega$ \\
$R_{L}$ & $7 \Omega$ \\
$k$ & 0.0001 to 0.1 \\
AC voltage & $30 \mathrm{~V}$ \\
AC frequency & $620 \mathrm{kHz}$ to $680 \mathrm{kHz}$ \\
\hline
\end{tabular}

[14]. For a given coil system, the optimal loading capacitance and port impendence are calculated as

$$
\begin{gathered}
R_{s 1}=r_{1} \sqrt{1+\frac{(\omega M)^{2}}{r_{1} r_{2}}}, \\
R_{s 2}=r_{2} \sqrt{1+\frac{(\omega M)^{2}}{r_{1} r_{2}}}, \\
C_{1}=\frac{1}{\omega^{2} L_{1}}, \\
C_{2}=\frac{1}{\omega^{2} L_{2}},
\end{gathered}
$$

where $R_{s 1}$ is the sum of $r_{p}$ and $R_{c 1}$. $R_{s 2}$ is the sum of $R_{c 2}$ and $R_{L}$. Equations (1) and (2) represent the resistance matching condition and (3) and (4) depict the resonance condition. From (3) and (4), it can be seen that in order to maximize the power transmission, the two coils on both sides are required to work at the resonant frequency as

$$
\omega_{0}=\sqrt{L_{1} C_{1}}=\sqrt{L_{2} C_{2}} .
$$

Theoretically, (1)-(4) are able to find out the optimum parameters elegantly. However, in practical applications, as $R c_{1}, R c_{2}$, and $r_{p}$ are parasitic values, there is little flexibility for engineers to actually choose circuit parameters for the resistance matching. For a given WPT system, $R c_{1}, R c_{2}, R_{p}$, and $R_{L}$ are fixed values, where it is not possible or convenient to change them.

2.2. Practical Circuit Model. An experimental test rig whose measured circuit parameters are listed in Table 1 is set up 
in the laboratory. Both $\mathrm{Tx}$ and $\mathrm{Rx}$ sides have the same inductance and capacitance, so they share the same resonant frequency as required in (5). However, for the resistance part, there is little flexibility for the parameter changes. All the resistance values such as $r_{1}, r_{2}, R_{c 1}$, and $R_{c 2}$ are the practical measurement from the test rig.

Normally the distance between the two coils can be several times of the coil radius, which makes the coupling coefficient $k$ a very small value around 0.001 to 0.01 . Only a small amount of the flux generated by Tx coil is able to go through the Rx coil. However, a large amount of magnetic energy can still be transferred through the limited amount of flux with relatively high efficiency (around 20 to 30\%) when the $\mathrm{Rx}$ and Tx coils are in the resonant state. This phenomenon can be explained by the circuit and magnetic theory.

The two-coil model depicted in Figure 1 can be analyzed using Kirchhoff's voltage law (KVL) as follows:

$$
\begin{aligned}
& I_{1}\left(R_{1}+j \omega L_{1}+\frac{1}{j \omega C_{1}}\right)+j \omega I_{2} M=V_{s}, \\
& I_{2}\left(R_{2}+j \omega L_{2}+\frac{1}{j \omega C_{2}}\right)+j \omega I_{1} M=0,
\end{aligned}
$$

where

$$
\begin{aligned}
& R_{1}=R_{c 1}+r_{1}+R_{p}, \\
& R_{2}=R_{c 2}+r_{2}+R_{L},
\end{aligned}
$$

and the $M$ is the mutual inductance between $\mathrm{Tx}$ and $\mathrm{Rx}$ coils. The relationship between the mutual inductance and coupling coefficient is defined as

$$
M=k \sqrt{L_{1} L_{2}} .
$$

In order to simplify the the two circuit equations (6) and (7) $Z_{1}$ and $Z_{2}$ are defined as the impendence of both circuit loops as follows:

$$
\begin{aligned}
& Z_{1}=R_{1}+j \omega L_{1}+\frac{1}{j \omega C_{1}}, \\
& Z_{2}=R_{2}+j \omega L_{2}+\frac{1}{j \omega C_{2}} .
\end{aligned}
$$

The two KVL equations (6) and (7) can be solved as

$$
\begin{gathered}
I_{1}=\frac{Z_{2} V_{s}}{Z_{1} Z_{2}+\omega^{2} M^{2}}, \\
I_{2}=-\frac{j \omega M_{12} V_{s}}{Z_{1} Z_{2}+\omega^{2} M^{2}} .
\end{gathered}
$$

Therefore, the input power $P_{I}$ which energizes the Tx coil and output power $P_{O}$ which are consumed on the load $R_{L}$ can be calculated as

$$
P_{I}=\left|V_{s} I_{1}\right| \cos \left(\angle V_{s}-\angle I_{1}\right), \quad P_{O}=\left|R_{L} I_{2}^{2}\right| .
$$

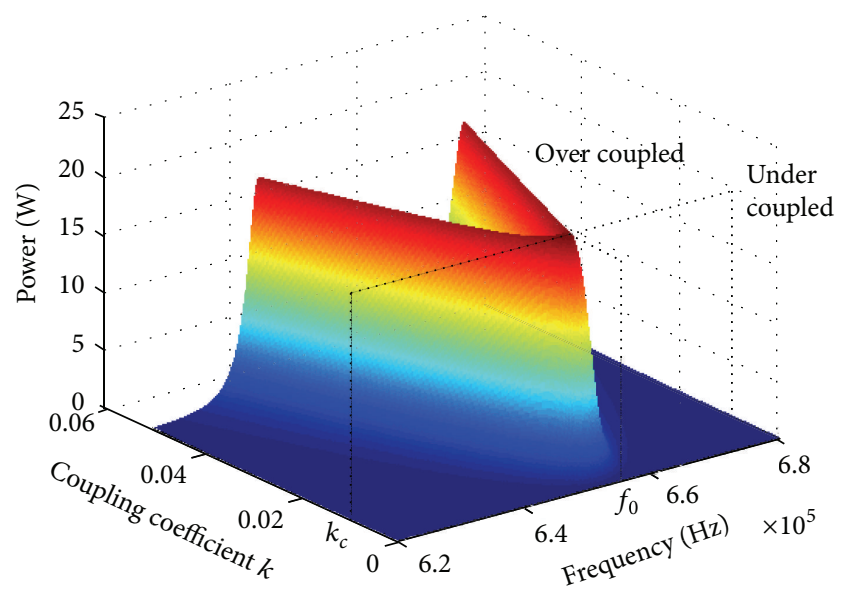

FIGURE 2: Output power $P_{O}$ as a function of frequency and coupling coefficient $k$ using the parameter given in Table 1 .

The overall WPT efficiency is

$$
E=\frac{P_{O}}{P_{I}}=\frac{R_{L} I_{2}^{2}}{\left|V_{s} I_{1}\right| \cos \left(\angle V_{s}-\angle I_{1}\right)} .
$$

Using data in Table 1, the output power against the exciting frequency and coupling coefficient can be plotted in Figure 2. When the coil distance is small and coupling coefficient between the two coils is big, the WPT works in the over coupled regime as marked in Figure 2. Frequency splitting can be clearly observed as there are two peak output powers. When the coupling between the two coils decreases with the increment of the distance, the system goes into the under coupled regime. The frequency separation between the two peak values decreases until they converge at a single frequency $f_{s}=f_{0} /\left(1-k^{2}\right)^{1 / 4}$, where $f_{0}$ is the resonant frequency of both coils [14].

2.3. Analysis of Practical Parameters. Due to the relative long distance between the two coils, the coupling efficient $k$ is very small ( 0.001 to 0.01$)$. Therefore, $f_{0}$ can be considered as a close approximation of $f_{s}$.

In practical applications, without loss of too much accuracy, technically it can be considered the two-split frequency merged at the resonant frequency $f_{0}$ when the coupling is $k_{c}$ as marked in Figure 2. For a practical WPT system with high $Q$ coils, $k_{c}$ can be considered as the critical point between the over coupled and under coupled regimes.

For practical system, the circuit parameters for $\mathrm{Tx}$ and $\mathrm{Rx}$ coils need to be aligned in order to get the maximum WPT power and efficiency, which means that

$$
L_{1}=L_{2}, \quad C_{1}=C_{2}, \quad \omega_{c 1}=\omega_{c 2},
$$

where $\omega_{c 1}$ and $\omega_{c 2}$ are the angular resonant frequency for the $\mathrm{Tx}$ and Rx coils, respectively. The resistance on the Rx side is normally bigger than that on the Tx side as the load resistance is connected in serial with the coil circuits. 


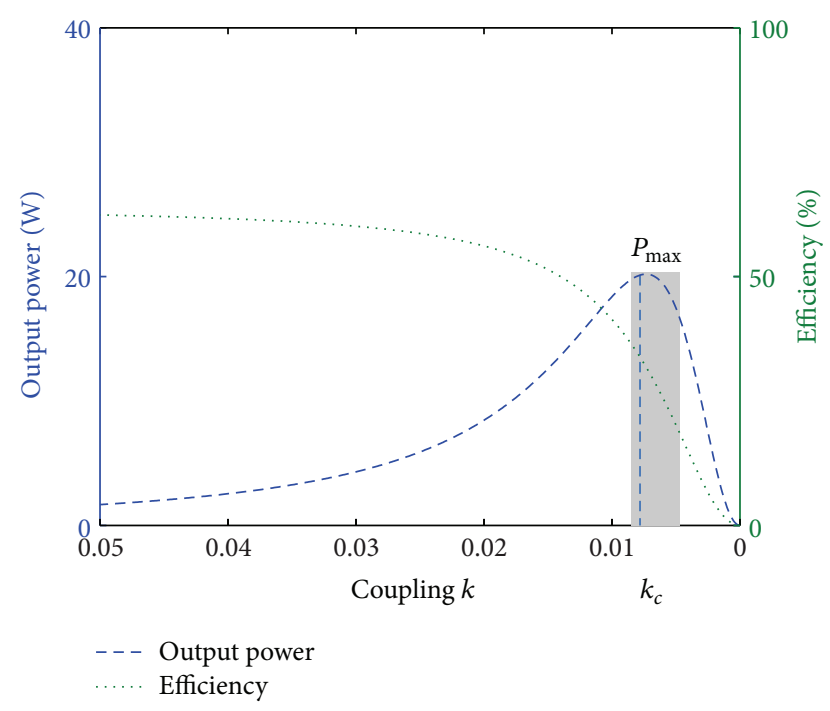

Figure 3: Output power and efficiency when $f=f_{0}$.

From the engineering point of view, $f_{0}$ can be used as a very close approximation to $f_{s}$ without inducing big calculation errors. Therefore, the WPT system at resonant frequency can be analyzed to find out the optimal operating area. When $f=f_{0}$, both the power $P_{O}$ and efficiency $E$ against coupling $k$ are plotted in Figure 3. It can be seen that the overall efficiency drops when the coupling $k$ is decreased. However, the output power increases with the decreasing coupling $k$ at the beginning until it reaches the peak value $P_{\max }$. Then it drops dramatically with the decrease of the coupling.

To simplify the analysis, it is assumed that both the Tx and $\mathrm{Rx}$ loops have the same inductance and capacitance as $L=L_{1}=L_{2}$ and $C=C_{1}=C_{2}$. The resonant frequency for both of the two coils is

$$
\omega_{0}=\frac{1}{\sqrt{L C}} .
$$

When $\omega=\omega_{0}$, combining (11), (12), and (15), the output power can be obtained as

$$
\left.P_{\mathrm{O}}\right|_{\omega=\omega_{0}}=R_{L} \frac{(1 / C) k^{2} L V_{s}^{2}}{\left(R_{1} R_{2}+(1 / C) k^{2} L\right)^{2}} .
$$

In order to get corresponding value of coupling $k_{c}$ at the maximum power, the derivative of (16) is taken with respect to $k$. By setting the result to zero and solving the equation, $k_{c}$ is obtained as

$$
k_{c}=\sqrt{\frac{R_{1} R_{2} C}{L}} .
$$

For the two coils, their series quality factor is defined as

$$
Q_{1}=\frac{1}{R_{1}} \sqrt{\frac{L}{C}}, \quad Q_{2}=\frac{1}{R_{2}} \sqrt{\frac{L}{C}} .
$$

Combining (17) and (18), the relationship between $k_{c}$ and the quality factors is

$$
k_{c}=\frac{1}{\sqrt{Q_{1} Q_{2}}} \text {. }
$$

The coupling $k$ is roughly reversely proportional to the third power of the coil distance. In order to get the maximum power at long distance, $k_{c}$ should be as small as possible, which means that $Q$ factors of both coils are very important parameters for the performance of the WPT. However, high $Q$ coils are very difficult to make due to the limitation of coil size, material, and so forth. For the case that the distance is several times of the coil diameter, normally the operating area is around the critical point as shown in the shadowed area in Figure 3.

\section{Practical Implementation of WPT Systems}

There are many existing WPT systems which successfully demonstrated the potential of the new technology in recent years. However, most of them are working with very high frequency around $10 \mathrm{MHz}$ which is far beyond the capacity of the normal switching power electronics such as IGBTs and MOSFETs. Therefore, for a WPT system which can be easily manufactured using the normal power electronic components, the switching frequency must be lower than $1 \mathrm{MHz}$ (for MOSFETs).

3.1. General Structure. Figure 4 is the diagram of the practical application of the proposed WPT system. A DDS (direct digital synthesizer) module AD9850 controlled by a MCU (microcontroller unit) controller is adopted to generate the accurate square wave exciting signal. The frequency signal is amplified by a gate driver module and then drives a MOSFET $\mathrm{H}$-bridge to generate a high frequency AC. The Tx coil is energized by the AC and transmits the power to the Rx coil though the inductive coupling. The high frequency $\mathrm{AC}$ is rectified to DC using bridge rectifier on the Rx side. The DC is filtered by capacitors before it drives the load.

3.2. Coil Design. In order to get a less than $1 \mathrm{MHz}$ resonant frequency for the Tx and Rx coils, both the inductance and capacitance need to be significantly bigger than the high frequency ones. The radius of the two coils is $20 \mathrm{~cm}$. They are winded by 19 turns using Litz cable with 500 strands, which forms inductance of $298 \mathrm{uH}$.

Low resonant frequency requires bigger capacitance as well as big inductance, which means the parasitic capacitance of the coil winding itself is not big enough and add-on capacitors connected in serial with the winding are necessary. From (12), it is clear that lower capacitance can increase the $Q$ factor of the circuit and improve the performance of the system. However, capacitors with small capacitance normally induce bigger parasitic resistance, which affects the $Q$ factor on an opposite way. In some cases, capacitors need to be connected in parallel to get smaller paralytic resistance at the expense of bigger capacitance.

Figure 5 is the circuit model for a practical coil design in which more than one high voltage ceramic capacitor is connected in parallel to decrease the overall parasitic resistance. The overall parasitic resistance is

$$
R_{\text {coil }}=\frac{1}{n} R_{c}+R_{L}
$$




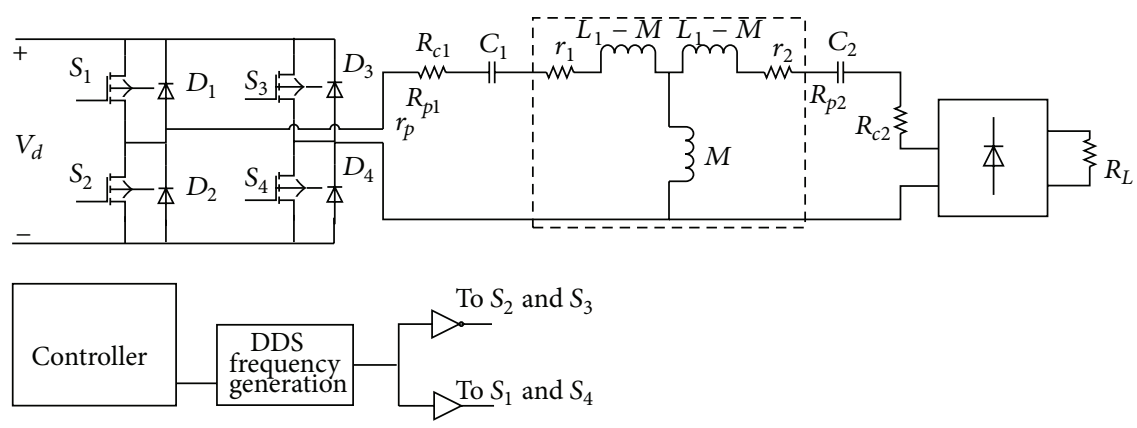

Figure 4: Diagram of the practical implementation.

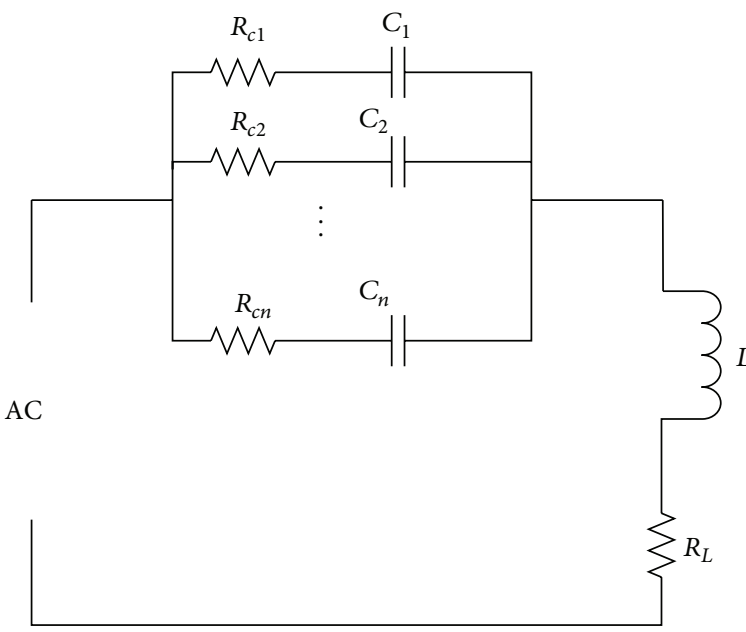

FIgURE 5: Circuit model for parallel connected capacitors.

where $R_{c}$ is the parasitic resistance for each individual capacitor and the overall capacitance is

$$
C_{\text {coil }}=n C \text {. }
$$

Therefore, the $Q$ factor of the coil can be calculated as

$$
Q_{\text {coil }}=\frac{1}{R_{\text {coil }}} \sqrt{\frac{L_{\text {coil }}}{C_{\text {coil }}}}=\frac{n}{R_{c}} \sqrt{\frac{L_{\text {coil }}}{n C+R_{L}}} .
$$

For a given type of capacitor, the number of the capacitors $n$ needs to be calculated and balanced in order to get the optimal $Q$ factor. In this case, two $100 \mathrm{pF}$ HV ceramic capacitors are connected in serial to get the best performance. Together with the $298 \mathrm{uH}$ winding inductance, the resonance frequency for both of the two coils is around $642 \mathrm{kHz}$.

3.3. Frequency Generation. Because of the high $Q$ factors of the coils, the WPT is very sensitive to the frequency drifting and jigging. A small variation of inciting frequency could result in significant performance drop which can be seen in Figure 2. Conventional analog signal generation cannot meet the precision requirement of the WPT system. Therefore, a digital method based on direct digital synthesizer (DDS) technology is selected as the solution.

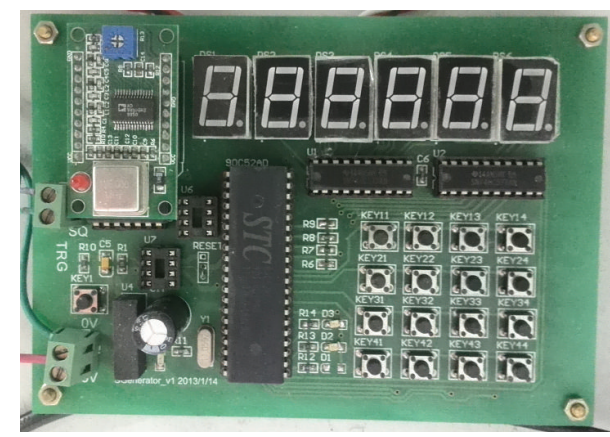

Figure 6: DDS frequency generation with MCU.

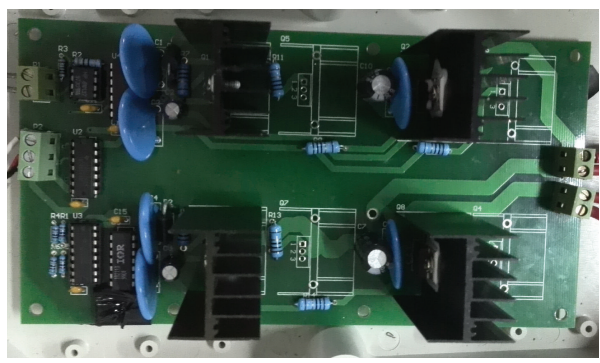

Figure 7: High frequency inverter.

A direct digital synthesizer (DDS) module AD9850 controlled by a MCU (microcontroller unit) as shown in Figure 6 is adopted to generate the accurate square wave exciting signal. Using the keyboard on the MCU controller, the output frequency can be tuned from $0.1-1 \mathrm{MHz}$ with the step size of $10 \mathrm{~Hz}$. The output square wave is highly stable compared with the analog circuit due to the explicit advantages of digital frequency generation.

3.4. High Frequency Inverter and Rectifier. The coil design makes the whole WPT system switch at $642 \mathrm{kHz}$ which is within the range of Ultra-fast MOSFET. A MOSFET $\mathrm{H}$-bridge is built to amplify the high frequency signal generated by the DDS module and energizes the Tx coil to transmit the electric power. 


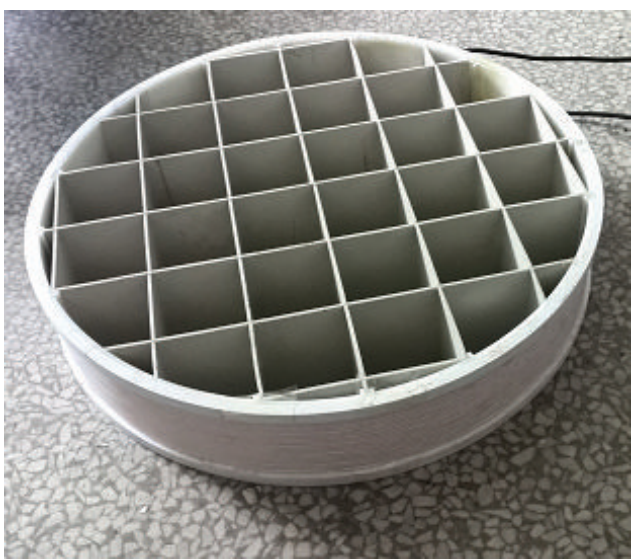

(a)

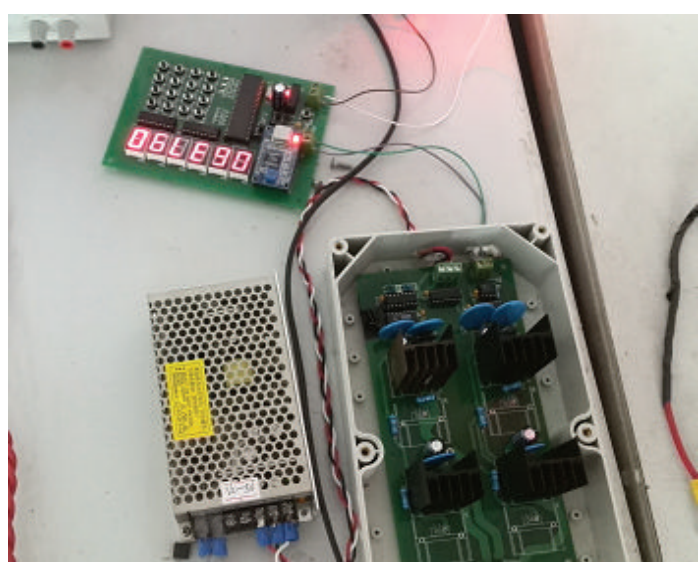

(b)

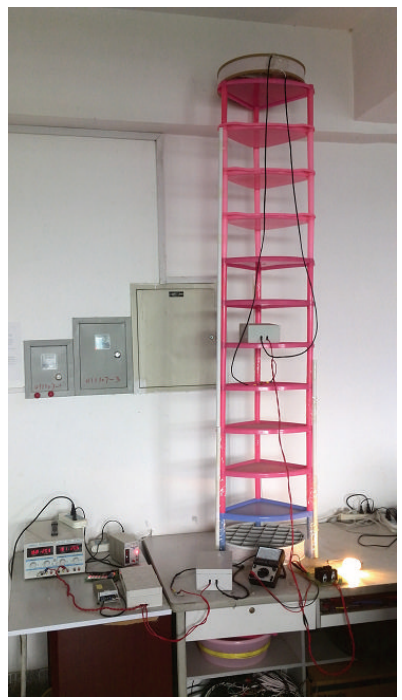

(c)

FIgure 8: Picture of the WPT system. (a) Coil structure. (b) Power electronics. (c) Test rig.

Assuming the DC input of the inverter is $V_{s}$ and using Fourier expansion, the square wave $\mathrm{AC}$ output put can be represented in infinite serials of the form

$$
\begin{aligned}
& V_{\text {square }} \\
& \qquad=\frac{4 V_{s}}{\pi}\left(\sin (2 \pi f t)+\frac{1}{3} \sin (6 \pi f t)+\frac{1}{5} \sin (10 \pi f t) \cdots\right),
\end{aligned}
$$

where the square wave is transformed into a serial of sinusoidal waves with different aptitudes and frequency.

From Figure 2, it can be seen that only the signals a very narrow band of the frequency are able to pass through the WPT system and the gain for other frequencies is significantly low. The WPT system actually operates like a very sensitive band pass filter. Even though the H-bridge inverter can only generate square waves, only the fundamental frequency is able to go through and other parasitic frequencies are cut off. Therefore, the theoretical analysis based on the AC source is still applicable for the case of $\mathrm{H}$-bridge inverters.
Figure 7 is the picture of the inverter in which four Ultrafast MOSFET IRF740s forms the H-bridge. The H-bridge is driven by two half-bridge gate driver IR2110. IR2110 has a build-in dead-band of $10 \mathrm{~ns}$. It is expanded to $50 \mathrm{~ns}$ to prevent the possible shoot-though because of the high speed switching. On the Rx side, due to the high working frequency, conventional diodes are not fast enough to rectify high frequency AC without inducing significant losses. Therefore, the electric energy received by the coil is rectified to DC by a high speed bridge rectifier made of Shockley diodes. Shockley diodes have very small reverse recovery time (less than $50 \mathrm{~ns}$ ), which reduces the loss greatly at the high working frequency.

\section{Experimental Results}

The radius of the both coils is $20 \mathrm{~cm}$ and the distance between the two coils is $2.2 \mathrm{~m}$. On the $\mathrm{Rx}$ side, a bulb which is selected as the load is illuminated using the energy received from the magnetic resonance as shown in Figure 8. 
TABLE 2: Circuit parameters.

\begin{tabular}{lcccc}
\hline Frequency & Load voltage & Load current & Power & Efficiency \\
\hline $638 \mathrm{kHz}$ & $6 \mathrm{~V}$ & $0.83 \mathrm{~A}$ & $5.0 \mathrm{~W}$ & $13.2 \%$ \\
$639 \mathrm{kHz}$ & $10 \mathrm{~V}$ & $1.39 \mathrm{~A}$ & $13.9 \mathrm{~W}$ & $23.8 \%$ \\
$640 \mathrm{kHz}$ & $11.9 \mathrm{~V}$ & $1.65 \mathrm{~A}$ & $19.6 \mathrm{~W}$ & $20.3 \mathrm{~W}$ \\
$641 \mathrm{kHz}$ & $12.1 \mathrm{~V}$ & $1.68 \mathrm{~A}$ & $19.9 \mathrm{~W}$ & $28.9 \%$ \\
$642 \mathrm{kHz}$ & $12 \mathrm{~V}$ & $1.66 \mathrm{~A}$ & $20.3 \mathrm{~W}$ & $27.8 \%$ \\
$643 \mathrm{kHz}$ & $12.1 \mathrm{~V}$ & $1.68 \mathrm{~A}$ & $18.4 \mathrm{~W}$ & $27.5 \%$ \\
$644 \mathrm{kHz}$ & $11.5 \mathrm{~V}$ & $1.60 \mathrm{~A}$ & $11.3 \mathrm{~W}$ & $26.5 \%$ \\
$645 \mathrm{kHz}$ & $9 \mathrm{~V}$ & $1.25 \mathrm{~A}$ & $4.2 \mathrm{~W}$ & $22.4 \%$ \\
$646 \mathrm{kHz}$ & $5.5 \mathrm{~V}$ & $0.76 \mathrm{~A}$ & & $9.6 \%$ \\
\hline
\end{tabular}

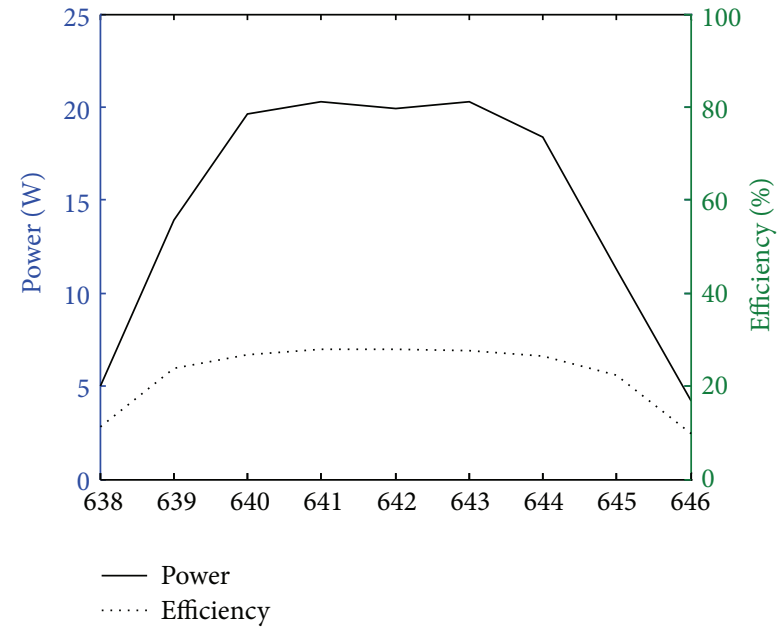

FIGURE 9: Experimental results.

The power supply for WPT prototype is a $40 \mathrm{~V} 5 \mathrm{~A}$ DC power source. The resonant frequencies of the Tx and $\mathrm{Rx}$ coils are well matched by adjusting both circuits carefully. The input power, load voltage, and load current are measured. Therefore, both the output power and overall efficiency (with the loss on the power electronics) can be calculated. The experimental results are shown in Table 2. Both the output power and efficiency against the frequency are plotted in Figure 9. It can be seen that high transfer efficiency (more than $20 \%$ ) can be maintained for a quite wide range from $639 \mathrm{kHz}$ to $645 \mathrm{kHz}$.

Because the coupling is bigger than the critical value $k_{c}$, the output power does not reach the maximum at the resonant frequency when the resonant frequencies are well aligned. The frequency splitting can be observed clearly as there are two peaks for the output power which are placed on both sides of $f_{0}$, which is indicated from the circuit analysis in Section 2.

\section{Conclusion and Future Work}

In this paper, the circuit modelling and implementation of a practical 2-coil WPT system have been introduced. Using the circuit theory, the relationship between the circuit parameters, coupling coefficient power transfer, and efficiency are analyzed. The optimal working area balancing the efficiency and distance is recommended based on the analysis. In order to verify the WPT proposed circuit theories, a practical WPT prototype is designed and implemented in this paper. The detailed structure of the prototype including the Tx and $\mathrm{Rx}$ coils, frequency generation, and high frequency power electronics has also been explained in this paper.

Using the prototype, the WPT experiments at the distance of $2.2 \mathrm{~m}$ are conducted. The experiments results show that the WPT prototype can achieve $20 \mathrm{~W}$ power transfer at relative high overall efficiency (more than 20\%). The voltage splitting phenomenon can be clearly observed as it is predicted from the theoretical analysis.

Since all the components utilized to build the prototype are made of normal electric and electronics components which can be purchased easily from the market with very low costs, there is great potential for the prototype to be commercialized in the future.

\section{Conflict of Interests}

The authors declare that there is no conflict of interests regarding the publication of this paper.

\section{Acknowledgment}

This research was supported by the National Science Foundation of China under Grant 61374064 and 61304152.

\section{References}

[1] A. P. Sample, D. J. Yeager, P. S. Powledge, A. V. Mamishev, and J. R. Smith, "Design of an RFID-based battery-free programmable sensing platform," IEEE Transactions on Instrumentation and Measurement, vol. 57, no. 11, pp. 2608-2615, 2008.

[2] A. Kurs, A. Karalis, R. Moffatt, J. D. Joannopoulos, P. Fisher, and M. Soljacic, "Wireless power transfer via strongly coupled magnetic resonances," Science, vol. 317, no. 5834, pp. 83-86, 2007.

[3] A. Karalis, J. D. Joannopoulos, and M. Soljačić, "Efficient wireless non-radiative mid-range energy transfer," Annals of Physics, vol. 323, no. 1, pp. 34-48, 2008. 
[4] R. E. Hamam, A. Karalis, J. D. Joannopoulos, and M. Soljačić, "Efficient weakly-radiative wireless energy transfer: an EIT-like approach," Annals of Physics, vol. 324, no. 8, pp. 1783-1795, 2009.

[5] Z. N. Low, R. A. Chinga, R. Tseng, and J. Lin, "Design and test of a high-power high-efficiency loosely coupled planar wireless power transfer system," IEEE Transactions on Industrial Electronics, vol. 56, no. 5, pp. 1801-1812, 2009.

[6] L. Chen, S. Liu, Y. C. Zhou, and T. J. Cui, "An optimizable circuit structure for high-efficiency wireless power transfer," IEEE Transactions on Industrial Electronics, vol. 60, no. 1, pp. 339-349, 2013.

[7] M. Kiani and M. Ghovanloo, "The circuit theory behind coupled-mode magnetic resonance-based wireless power transmission," IEEE Transactions on Circuits and Systems. I. Regular Papers, vol. 59, no. 9, pp. 2065-2074, 2012.

[8] J. de Boeij, E. Lomonova, J. L. Duarte, and A. J. A. Vandenput, "Contactless power supply for moving sensors and actuators in high-precision mechatronic systems with long-stroke power transfer capability in x-y plane," Sensors and Actuators A: Physical, vol. 148, no. 1, pp. 319-328, 2008.

[9] P. Li and R. Bashirullah, "A wireless power interface for rechargeable battery operated medical implants," IEEE Transactions on Circuits and Systems II: Express Briefs, vol. 54, no. 10, pp. 912-916, 2007.

[10] P. Si, A. P. Hu, S. Malpas, and D. Budgett, "A frequency control method for regulating wireless power to implantable devices," IEEE Transactions on Biomedical Circuits and Systems, vol. 2, no. 1, pp. 22-29, 2008.

[11] J. L. Villa, J. Sallán, A. Llombart, and J. F. Sanz, "Design of a high frequency inductively coupled power transfer system for electric vehicle battery charge," Applied Energy, vol. 86, no. 3, pp. 355-363, 2009.

[12] S. Jones, G. Bucea, A. McAlpine et al., "Condition monitoring system for TransGrid 330kV power cable," in Proceedings of the International Conference on Power System Technology (POWERCON '04), pp. 1282-1287, Singapore, November 2004.

[13] E. Gulski, P. Cichecki, J. J. Smit, P. P. Seitz, B. Quak, and F. de Vries, "On-site condition monitoring of HV power cables up to $150 \mathrm{kV}$," in Proceedings of the International Conference on Condition Monitoring and Diagnosis (CMD '08), pp. 1199-1202, April 2008.

[14] N. Inagaki, "Theory of image impendence matching for inductively coupled power transfer systems," IEEE Transactions on Microwave Theory and Techniques, vol. 62, pp. 901-908, 2014.

[15] R. Roberts, "Conjugate-image impendences," Proceeding of the IRE, vol. 34, no. 4, pp. 198-204, 1946.

[16] M. Kiani, U.-M. Jow, and M. Ghovanloo, "Design and optimization of a 3-coil inductive link for efficient wireless power transmission," IEEE Transactions on Biomedical Circuits and Systems, vol. 5, no. 6, pp. 579-591, 2011.

[17] A. P. Sample, D. A. Meyer, and J. R. Smith, "Analysis, experimental results, and range adaptation of magnetically coupled resonators for wireless power transfer," IEEE Transactions on Industrial Electronics, vol. 58, no. 2, pp. 544-554, 2011.

[18] T. C. Beh, M. Kato, T. Imura, S. Oh, and Y. Hori, "Automated impedance matching system for robust wireless power transfer via magnetic resonance coupling," IEEE Transactions on Industrial Electronics, vol. 60, no. 9, pp. 3689-3698, 2013. 

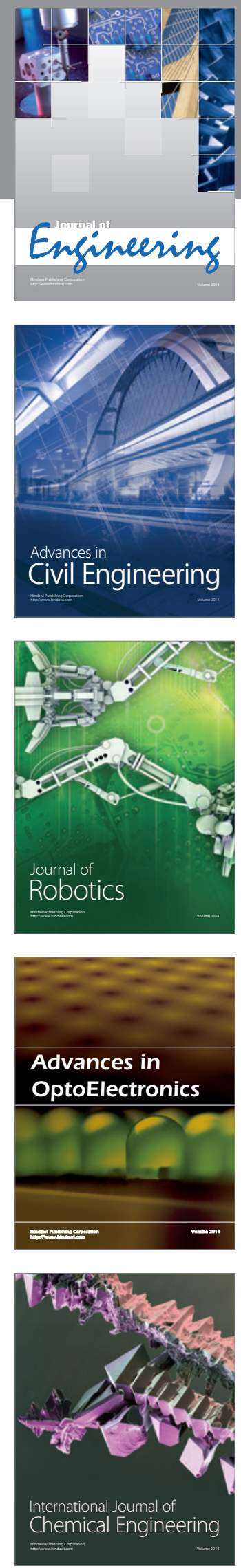

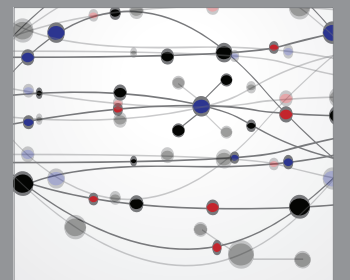

The Scientific World Journal
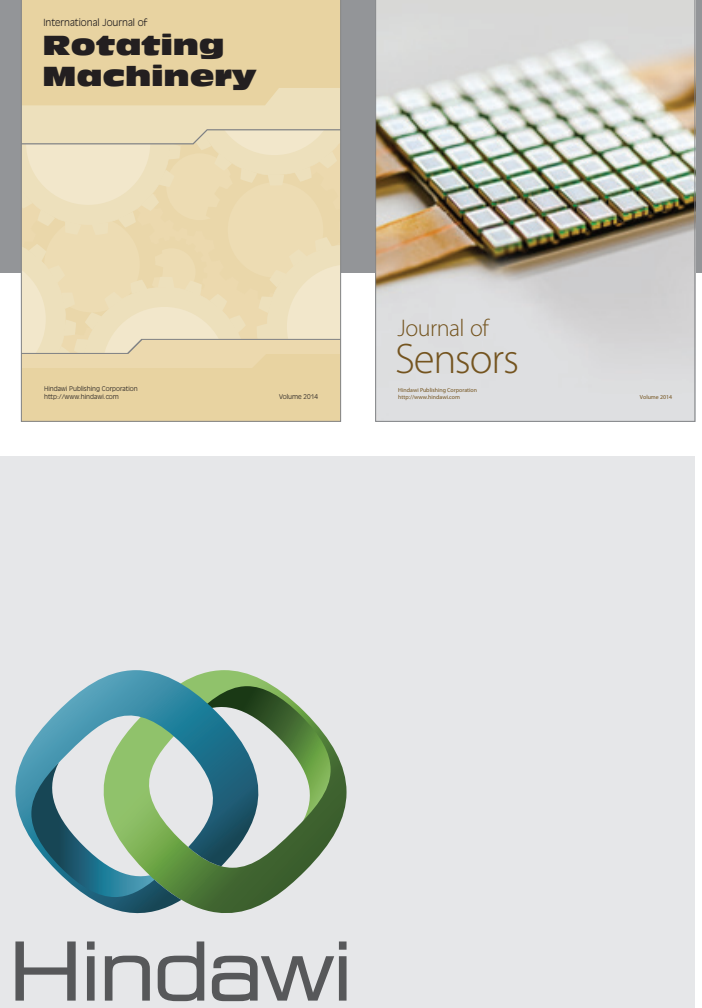

Submit your manuscripts at http://www.hindawi.com
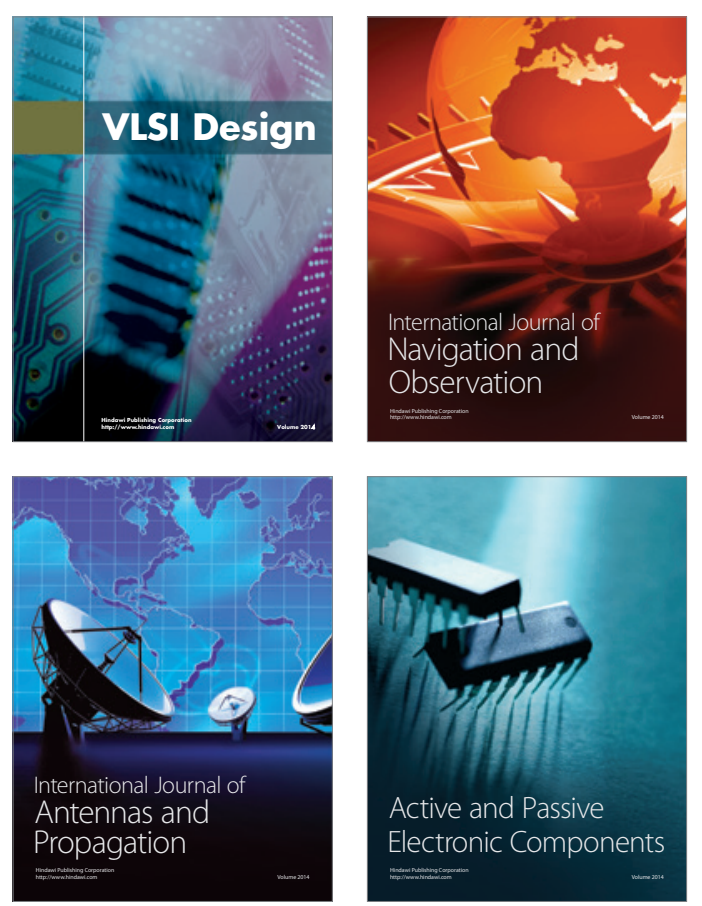
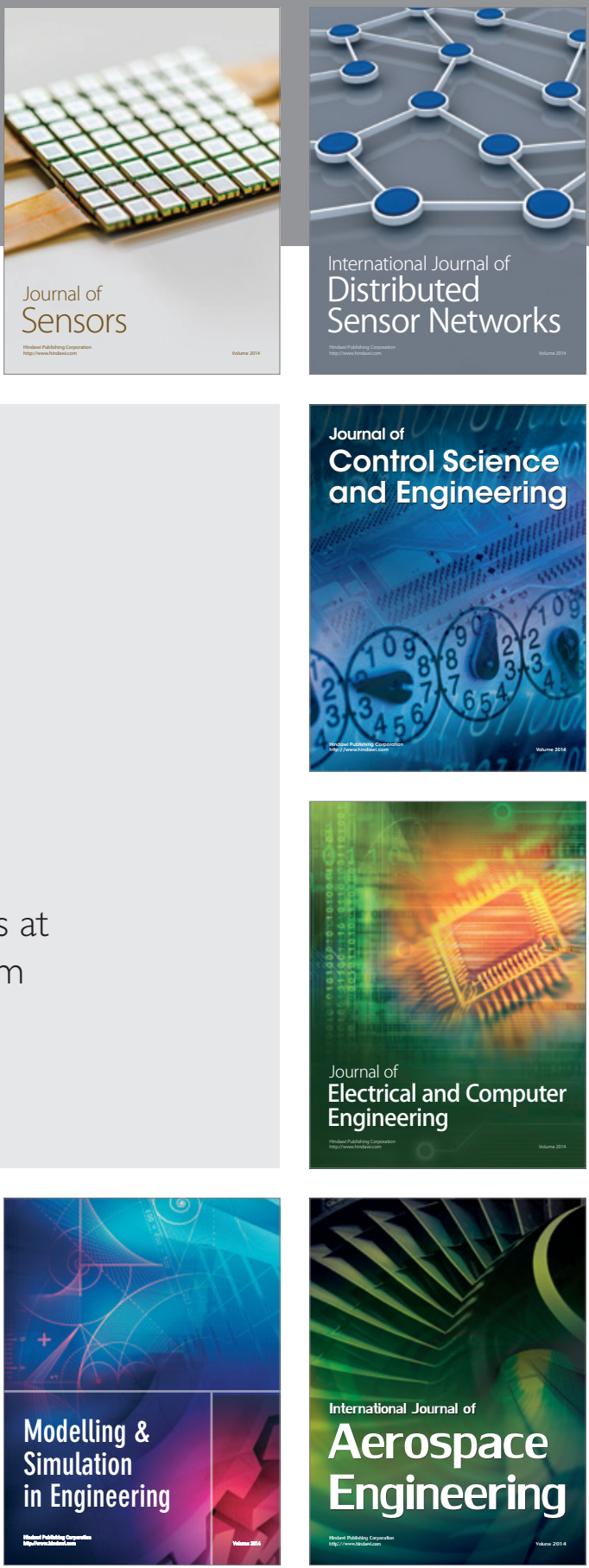

Journal of

Control Science

and Engineering
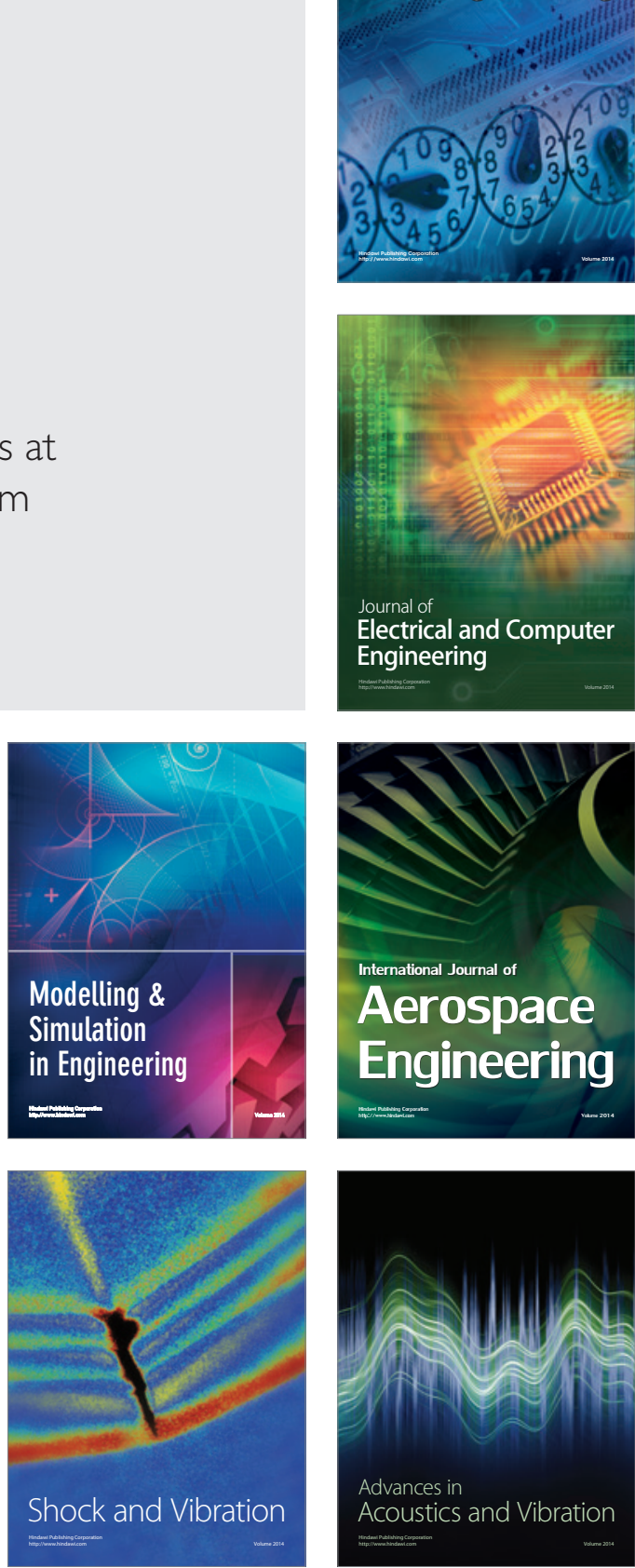